\title{
A longitudinal study of clinical and immunological findings in 52 patients with relapsing retinal vasculitis
}

\author{
M R STANFORD,' ELIZABETH GRAHAM,'2 EVA KASP,' M D SANDERS, \\ AND D C DUMONDE'
}

From the Departments of 'Immunology and ${ }^{2}$ Medical Ophthalmology, United Medical and Dental Schools, St Thomas's Campus, London SE1 7EH

SUMMARY Fifty-two patients with retinal vasculitis-26 with idiopathic disease and 26 with associated systemic inflammatory disease-were followed up for periods ranging from six months to 12 years. The aim of the study was to determine the relationship between relapse of uveitis, visual outcome, and the occurrence of circulating immune complexes (CIC) and antiretinal antibodies. In a total of 69 relapses, CIC were increased in one-third of patients and antiretinal antibodies in one-half. In those 34 patients who expressed antiretinal antibodies $27(79 \%)$ of the relapses were characterised by antiretinal antibodies in the absence of raised CIC levels $(\mathrm{p}<0 \cdot 01)$. These findings support our previous hypothesis that CIC may have a protective role in autoimmune retinal vasculitis and that antiretinal autoimmunity is of pathogenetic importance in relapse. In individual patients the visual outcome was not related to the number of relapses or to the CICautoantibody pattern, suggesting the operation of additional features which merit identification.

Retinal vasculitis is a significant cause of blindness in young people. Clinical diagnosis depends on evidence of inflammation within the eye (for example, cells in the vitreous), together with involvement of the retinal vessels (for example, periphlebitis, vascular occlusion, or fluorescein leakage). The main causes of visual loss are macular oedema and retinal vascular occlusion occasionally leading to neovascularisation and vitreous haemorrhage. Retinal vasculitis may be either idiopathic or associated with systemic inflammatory disease (for example, sarcoidosis, Behçet's disease). Both cell mediated and humoral immunity to specific retinal antigens together with raised circulating immune complex levels have been demonstrated in patients with retinal vasculitis of varying aetiologies. In a recent point-prevalence study ${ }^{1}$ we showed that $70 \%$ of all patients with retinal vasculitis expressed retinal autoimmunity, and that in patients with severe idiopathic retinal vasculitis there was an inverse relationship between pronounced antiretinal antibody levels and the occurrence of raised circulating immune complexes.

Correspondence to Dr E M Graham, Medical Ophthalmology Unit, St Thomas's Hospital, London SE1 7EH.
We present the results of a longitudinal study of 52 patients with relapsing retinal vasculitis which was carried out to see if these immunological markers could also predict the course and outcome of disease.

\section{Materials and methods}

Fifty-two patients were recruited into the study from those attending the Medical Eye Unit at St Thomas's Hospital. The majority of these patients had been diagnosed elsewhere and referred for assessment, the rest being admitted from the Casualty Department. In all cases the diagnosis was confirmed by the clinical and fluorescein angiographic appearances. The patients had all been followed up regularly (2-3 month assessments) for a minimum of six months. Twenty-six patients had retinal vasculitis alone (RV), and 26 had retinal vasculitis in association with systemic inflammatory disease (RV+SID). In the latter group 22 patients had Behçet's disease, three had sarcoidosis, and one had juvenile polyarthritis. Patients with infective or malignant disease, or whose follow-up was less than six months, were excluded from the study. Previous treatment was not a contraindication to inclusion.

A full ophthalmological assessment was carried 
out at each hospital visit and included tests of best corrected visual acuity, near vision, colour vision, slit-lamp examination, and indirect ophthalmoscopy. At most of his attendances the patient was seen by one ophthalmologist (EG). Fluorescein angiography was performed whenever clinically indicated. Disease severity was scored on a 20-point system in which specific ophthalmological features were weighted according to the considered impact on short-term visual morbidity. ${ }^{\prime}$

The course of the disease was described in terms of the number of relapses for each patient. A relapse was defined as an increase in visual symptoms (blurred vision, floaters) associated with an increase in vitreous cellularity. This was often associated with the appearance of one or more new features of retinal inflammation (macular oedema, retinal infiltrates, vascular occlusion). An individual relapse was frequently, though not always, associated with an increase in the disease severity score and with a reduction in visual acuity.

The outcome of disease was described in terms of overall changes in visual acuity during the study period. For the purposes of this study corrected visual acuity was graded as follows: 'excellent' (grade I: $6 / 6$ or better); 'good' (grade II: $6 / 9$ or $6 / 12$ ); 'fair' (grade III: 6/18 to 6/36); and bad (grade IV: 6/60 or worse). A good visual outcome was defined as either (a) maintenance of a visual acuity of $6 / 12$ or better; or (b) improvement of one or more grades by the end of the study period. Patients who did not fulfil either of these criteria were considered to have had a poor visual outcome.

Patients were treated with combinations of topical therapy (steroids, mydriatics), systemic corticosteroids, and immunosuppressives (for example, azathioprine, chlorambucil, colchicine, and cyclosporin A). In general, patients with a visual acuity of less than $6 / 12$ were treated systemically at a starting dose of prednisolone $60 \mathrm{mg} / \mathrm{day}$, with an immunosuppressive agent being added after six weeks if the disease did not remit.

Blood was taken from all patients at each visit for immunological assessment.

IMMUNOLOGICAL ASSESSMENT

Circulating immune complex (CIC) measurements and complement screening tests

CIC were measured by two methods: (a) Serum was precipitated with $2 \%$ polyethylene glycol (PEG), and the IgG content of the precipitate was measured by radial immunodiffusion. (b) Serum was reacted with solid phase human $\mathrm{C1q}$. Immune complexes were then determined by reactivity with a ${ }^{125}$ I-labelled antiglobulin reagent, the test being calibrated with aggregated human IgG. The sensitivity of the method was increased by preincubating Clq-coated tubes with non-labelled rabbit IgG antibody directed against the Fc fragment of human IgG. This method reduced the prevalence of false negative results in sera containing rheumatoid factor.

Complement screening tests were done on all sera, with haemolytic plates for testing the functional activity of classic and alternative pathways and radial immunodiffusion for measuring components $\mathrm{C} 3$ and C4. CIC levels were considered to be raised when C1q or PEG results were more than 2 SD above the normal mean (for $\mathrm{C} 1 \mathrm{q}: 14 \cdot 2 \pm 1 \cdot 2 \mathrm{mg} / \mathrm{l}$ equivalent aggregated IgG; for PEG: $>4.9 \mathrm{~g} / \mathrm{l}$ equivalent IgG). An abnormal complement screen was recorded when the result of any of the four tests fell outside the normal range.

\section{Retinal specific autoantibodies}

Antiretinal antibodies were measured by two methods: (a) Passive haemagglutination of sheep erythrocytes to which bovine $S$ antigen had been coupled with the aid of chromium chloride. (b) Indirect immunofluorescent staining of frozen sections of human retina. The specificity of antiretinal immunofluorescence was established by the use of a reference standard guinea-pig antiserum, raised against photoreceptor antigen, which did not contain antinuclear antibodies or antibodies to smooth muscle. Retinal autoantibodies were considered to be present if fluorescence was found in the photoreceptor layers of cones and rods and the cytoplasm of the outer nuclear and outer plexiform layers. As sera from healthy controls occasionally gave antiretinal immunofluorescence at 1:5 dilution, or a passive haemagglutination titre of $1: 16$, positive results were recorded when antibodies were detected at titres of $>1: 10$ by immunofluorescence or $>1: 32$ by haemagglutination.

Statistical analysis. All results in this study were analysed by $\chi^{2}$ test.

\section{Results}

Fifty-two patients who had been followed up for six months or more were included in this study-26 patients with RV alone and 26 with RV+SID.

Patients in the RV group were 13 to 70 years old (mean 37 years), attended for six to 56 months (mean 25 months), and had immunological assessments on two to 14 occasions (mean seven). Patients in the RV+SID group were 19 to 63 years old (mean 36), attended for six to 45 months (mean 18 months), and had immunological assessments on two to 25 occasions (mean nine). In all, individual ophthalmological and immunological assessments were made in these patients on 449 occasions. 
Table 1 Incidence of relapse in 52 patients with retinal vasculitis

\begin{tabular}{lcc}
\hline & $\begin{array}{l}R V \\
(26 \text { patients })\end{array}$ & $\begin{array}{l}R V+S I D \\
(26 \\
\text { patients })\end{array}$ \\
\hline 0relapse & 6 & 3 \\
1 relapse & 8 & 12 \\
2 relapses & 10 & 10 \\
3 relapses & 2 & 1 \\
Total no. of relapses & 34 & 35 \\
\hline
\end{tabular}

Table 2 Immunological findings during 69 relapses in all 52 patients

\begin{tabular}{lll}
\hline & Ret $A B+$ & $\operatorname{Ret} A B-$ \\
\hline CIC + & $7(10 \%)$ & $16(23 \%)$ \\
CIC - & $27(39 \%)$ & $19(28 \%)$ \\
Total & 34 & 35 \\
\hline
\end{tabular}

$\chi^{2}$ test, $\mathrm{p}=>0.05$.

\section{RELAPSES}

The number of relapses for each patient in both groups is shown in Table 1 . The 26 patients in the $R V$ group had a total of 34 relapses and the 26 patients in the RV+SID group had 35 relapses. At the time of relapse the CIC concentrations were raised in $38 \%$ of patients with RV and $31 \%$ of patients with $R V+S I D$, and antiretinal antibodies were found in $68 \%$ of patients with RV and $66 \%$ of patients with RV+SID. These features alone did not distinguish the two groups of patients. When both tests were examined together (Table 2), $28 \%$ of patients showed neither raised CIC nor retinal autoantibodies; one-third did have high CIC but associated with retinal antibodies in only $10 \%$. In contrast, $39 \%$ of patients who did not have high $\mathrm{CIC}$ did express retinal autoimmunity (p>0.05).

The relationship between CIC and retinal autoantibodies during relapse, which excludes patients not expressing immunological abnormalities, is shown in Table 3 . In patients with RV five relapses

Table 3 Relationship between CIC and retinal autoimmunity during relapses in patients expressing immunological abnormalities

\begin{tabular}{llllll}
\hline & \multicolumn{3}{l}{ CICpositive } & & \multicolumn{2}{l}{ Ret.ab. positive } \\
\cline { 2 - 3 } \cline { 5 - 6 } & $\begin{array}{l}\text { Ret. ab. } \\
\text { positive }\end{array}$ & $\begin{array}{l}\text { Ret. ab. } \\
\text { negative }\end{array}$ & & $\begin{array}{l}\text { CIC } \\
\text { positive }\end{array}$ & $\begin{array}{l}\text { CIC } \\
\text { negative }\end{array}$ \\
\hline RV & 5 & 8 & & 11 \\
RV+SID & 2 & 8 & & 2 & 16 \\
Total & 7 & 16 & 7 & 27 \\
\hline
\end{tabular}

Ret. ab. = retinal antibodies. CIC positive by either C1q or polyethylene glycol. Ret. ab. positive by either passive haemagglutination or indirect immunofluorescence tests. $\chi^{2}$ test, $\mathrm{p}<0 \cdot 001$.
Table 4 Immunological changes during quiescent disease

\begin{tabular}{crr}
\hline & $R V$ & $R V+S I D$ \\
\hline Ret. Ab. + & $82(48 \%)$ & $64(30 \%)$ \\
CIC + & $87(52 \%)$ & $147(70 \%)$ \\
- & $48(28 \%)$ & $54(25 \%)$ \\
\hline
\end{tabular}

Ret. ab. = retinal antibodies.

Table $5 \quad R V+S I D:$ Retinal autoantibodies and disease activity

\begin{tabular}{lcrr}
\hline & Quiescent & Active \\
\hline Ret. ab. + & 64 & 26 & 90 \\
Ret. ab. - & 147 & 95 & 156 \\
Total & 211 & & 246 \\
\hline & & \\
$\chi^{2}$ test, p<0.001 & \\
This table confirms that patients with RV +SID were much more \\
likely to show retinal autoimmunity during active phases of disease. \\
(There was not any similar correlation when CIC levels were \\
considered in relation to disease activity.)
\end{tabular}

(32\%) occurred when both CIC and retinal antibodies were present, but $11(58 \%)$ relapses occurred when retinal antibodies were present alone. Similarly in patients with RV+SID only two $(9 \%)$ relapses occurred when raised CIC and retinal autoantibodies were present, but $16(91 \%)$ with retinal autoantibodies alone $(\mathrm{p}<0.001)$. In RV $10(29 \%)$ relapses occurred in the absence of immunological abnormality and nine $(26 \%)$ in RV+SID.

There was no correlation between CIC levels and the number of relapses. In RV $6 \%$ of patients with two or more relapses and $7 \%$ of patients with one or no relapses had raised CIC. The figures for RV+SID were $8 \%$ and $9 \%$ respectively.

Patients were assessed clinically and immunologically 380 times when their disease was judged to be quiescent (RV, 169; RV+SID, 211: Table 4). At these inactive times CIC concentrations were raised in $28 \%$ of patients with $\mathrm{RV}$ and $25 \%$ of patients with $\mathrm{RV}+\mathrm{SID}$ and retinal autoantibodies in $48 \%$ of RV patients and $30 \%$ of RV+SID patients. In RV+SID retinal antibodies were significantly more prevalent in patients at the time of relapse (Table 5, p<0.001). There was no similar correlation in RV, and the presence of CIC did not distinguish the two phases of disease in either group. Complement screening did not show any abnormality in the two groups of patients.

Relapses were often associated with development of periphlebitis, vascular occlusion, macular oedema, or retinal infiltrates. Patients presented with periphlebitis on 60 occasions, which tended to occur with a rise in retinal autoantibodies. However, when patients presented with macular oedema, retinal autoimmunity was not usually expressed. 
Table 6 Relationship between visual outcome and relapse rate

\begin{tabular}{lcc}
\hline & Good visual outcome & Poor visual outcome \\
\hline$R V$ & 7 & 5 \\
High relapse rate & 5 & 9 \\
Low relapse rate & 12 & 14 \\
Total & & \\
$R V+S I D$ & 6 & 5 \\
High relapse rate & 4 & 11 \\
Low relapse rate & 10 & 16 \\
Total & & \\
\hline
\end{tabular}

\section{VISUAL OUTCOME}

Twelve patients with RV had a good visual outcome and 14 patients had a bad visual outcome. Similarly in RV+SID 10 patients had a good visual outcome and 16 a bad visual outcome.

There was no correlation between the number of relapses and the visual outcome in either group, though all three patients with three relapses had a bad visual outcome (Table 6).

In patients with $\mathrm{RV}$ the $\mathrm{CIC}$ concentrations were raised in $29 \%$ of patients with a good visual outcome and $27 \%$ patients with a bad visual outcome; retinal autoantibodies occurred in $48 \%$ of patients with good visual outcome and $52 \%$ patients with bad outcome.

In patients with $\mathrm{RV}+\mathrm{SID} 20 \%$ of patients with a good visual outcome had raised CIC concentrations and $29 \%$ patients with a bad visual outcome; $43 \%$ of patients with a good outcome and $33 \%$ of patients with a bad outcome showed retinal autoimmunity. Hence these abnormalities did not distinguish between a good or bad visual prognosis in either group of patients. When the number of relapses, visual outcome, and immunological status were examined together, no significant features were found to distinguish patients in any group.
Figs. 1 and 2 illustrate the recording of clinical and immunological progress of two patients with RV. Patient 1 , with a bad outcome, showed frequent retinal autoantibodies, particularly during relapses (high disease score) but generally not in association with raised CIC. Patient 2, with a good outcome, often had raised CIC during relapses, sometimes together with retinal antibodies, but did not express retinal autoimmunity in the absence of raised CIC.

\section{TREATMENT}

Twenty patients with $\mathrm{RV}$ required systemic treatment, and only six did not require any systemic or immunosuppressive treatment throughout the follow-up period. Only one patient with RV+SID did not require systemic treatment, all the remaining 25 requiring both steroids and immunosuppressives. Systemic treatment reduced the disease activity in all patients, but it was impossible to evaluate the effect of systemic therapy on immunological features because only three patients changed their mode of treatment during the follow-up period.

\section{Discussion}

The present study was undertaken to identify patterns of immunological behaviour that might predict visual prognosis in patients with relapsing retinal vasculitis. The study revealed that disease relapse is frequently associated with antiretinal antibodies unaccompanied by raised CIC levels. In idiopathic $\mathrm{RV}$ raised CIC levels did not distinguish an active phase from a quiescent phase of the disease. However, further examination of the patients with RV accompanying Behçet's disease showed that, although the level of antiretinal antibodies did not fluctuate during the course of retinal disease, raised $\mathrm{CIC}$ levels tended to occur just before or just after the

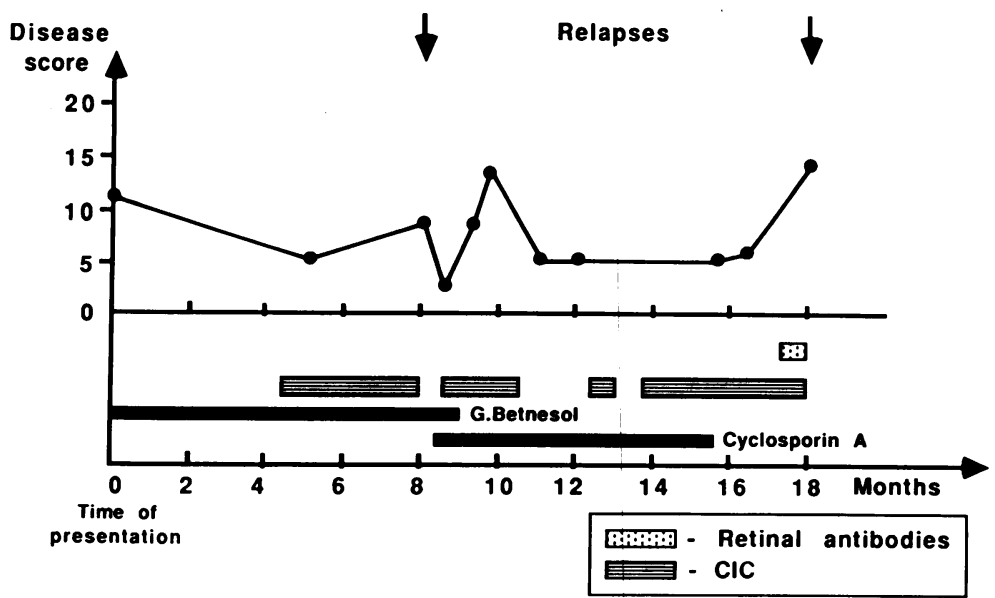

Fig. 1 Flow chart showing the clinical and immunological progress of a patient with retinal vasculitis and a bad visual prognosis. Antiretinal autoantibodies are raised prior to a relapse (arrows) in the absence of circulating immune complexes. G. Betnesol=betamethasone eyedrops. 
period of relapse. A similar observation was made by Nussenblatt $e t$ al. in patients receiving cyclosporin A for their Behçet's disease. ${ }^{2}$ On occasion raised CIC levels occurred during relapses in the absence of retinal autoimmunity; this suggests an immune complex mediated pathogenesis and was particularly observed in patients with Behçet's disease.

Our study did not show any correlation in either $\mathrm{RV}$ or RV+SID between relapse rate and visual prognosis, or between CIC, retinal autoimmunity, and visual prognosis.

The longitudinal relationship of CIC levels and retinal autoimmunity in posterior uveitis has been examined in three previous reports. ${ }^{3-5}$ Abrahams and Gregerson reported that there was no correlation between clinical activity and titres of antibody to $S$ antigen in four patients with relapses-three with idiopathic disease and one with sarcoidosis. ${ }^{4}$ In a further study ${ }^{3}$ it was reported that patients with only one attack of uveitis showed a gradual decrease in antiretinal antibody titres after the acute attack, while in patients with recurrent attacks of uveitis antiretinal antibody levels were generally stable. We could not confirm this observation in patients with $\mathrm{RV}+\mathrm{SID}$, though there was a trend towards it in patients with RV alone. Heckenlively et al. studied patients with non-inflammatory retinal disease and showed a significant correlation between antiretinal antibodies and various features on fluorescein angiography such as macular odema and disc staining. ${ }^{5}$

Antibodies to $\mathrm{S}$ antigen rise after argon laser therapy for diabetic retinopathy, which suggests that retinal destruction initiates an immune response and consequent antibody formation. ${ }^{6}$ This might lead one to expect an increase in antibody titres during the course of a disease such as Behçet's when relentless retinal damage occurs secondary to consecutive venous occlusions. However, we did not observe such a change in antibody titre. Retinal antibodies were measured in this study. by both passive haemagglutination and indirect immunofluorescence. In both tests the autoantigens are not fully specified: thus indirect immunofluorescence is undertaken with frozen sections of human retina that contain several potential autoantigens (S antigen, rhodopsin, interphotoreceptor binding protein). This heterogeneity of autoantigens may underlie the lack of correlation between disease severity and antibody levels in our study; related findings have been observed in other organ specific diseases such as myasthenia gravis. ${ }^{?}$

Immunological findings were not altered by changes in systemic therapy. The majority of patients were not taking systemic steroids when first examined, and we were unable to substantiate the findings of Gregerson et al. ${ }^{8}$ that systemic therapy reduces the levels of antibodies.

There is substantial evidence that retinal vasculitis is an immunologically mediated disease. The ocular inflammation often accompanies a systemic inflammatory disease of known immunological background, such as sarcoidosis and Behçet's disease. ${ }^{910}$ Sera from patients with retinal vasculitis may contain circulating immune complexes," or antibodies to retinal $\mathbf{S}$ antigen,' and their lymphocytes may show abnormal $\mathrm{T}$ cell responses to both standard mitogens and $S$ antigen. ${ }^{12}$ Despite this knowledge the pathogenesis of retinal vasculitis in any given patient remains elusive. However, this study lends support to our view that in isolated retinal vasculitis antiretinal
Fig. 2 Flow chart showing the clinical and immunological features of a retinal vasculitis patient with a good visual prognosis. The first relapse (arrows) is preceded by a rise in antiretinal antibody titre and its recovery with the development of CIC. A further attack is again associated with a raised antiretinal antibody titre in the absence of $\mathrm{CIC}$. G. Betnesol=betamethasone eyedrops. G. Maxidex= dexamethasone+hypromellose eyedrops.

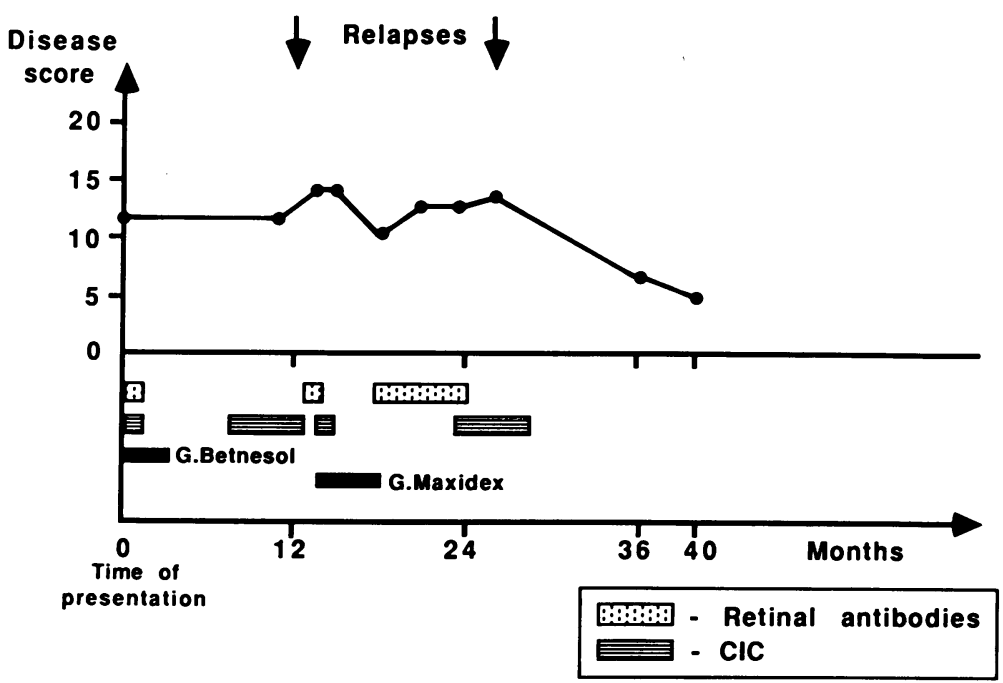


autoimmunity is pathogenic and that $\mathrm{CIC}$ may have a compensatory or protective role. ${ }^{13}$ In patients with RV+SID it appears that CIC may play a part in disease pathogenesis along with antiretinal antibodies in some patients.

We are indebted to Mr Richard Dewhirst for the illustrations and Miss Josephine Lace for secretarial assistance. This work was supported by grants from the Medical Research Council (Grant No. G.8303319 SB), the TFC Frost Charitable Trust, Research (Endowments) Committee, St Thomas's Hospital, the Frances and Augustus Newman Foundation, and the Iris Fund for Prevention of Blindness. Mr M R Stanford holds an MRC Training Award Fellowship.

\section{References}

1 Dumonde DC, Kasp Grochowska E, Graham E, et al. Antiretinal autoimmunity and circulating immune complexes in patients with retinal vasculitis. Lancet 1982; ii: 787-92.

2 Nussenblatt RB, Palestine AG, Chan C, Mochizuki M, Yancy K. Effectiveness of cyclosporin therapy for Behçet's disease. Arthtitis Rheum 1985; 28: 671-9.

3 Abrahams IW, Gregerson DS. Longitudinal study of serum antibody responses to retinal antigens in acute ocular toxoplasmosis. Am J Ophthalmol 1982; 93: 224-31.

4 Abrahams IW, Gregerson DS. Longitudinal study of serum antibody responses to bovine retinal $\mathrm{S}$ antigen in endogenous granulomatous uveitis. Br J Ophthalmol 1983; 67: 681-4.
5 Heckenlively JR, Solish AM, Chant SM, Meyers-Elliott RH. Autoimmunity in hereditary retinal degenerations. II. Clinical studies: antiretinal antibodies and fluorescein angiogram findings. Br J Ophthalmol 1985; 69: 758-64.

6 Gregerson DS, Abrahams WI, Puklin JE. Serum antibody responses to bovine retinal $S$ antigen and rod outer segments in proliferative diabetic retinopathy before and after argon laser photocoagulation. Ophthalmology 1982; 89: 767-71.

7 Roses AD, Alonow CW, McAdams MW, Lane RJM. No direct correlation between serum antiacetylcholine receptor antibody levels and clinical state of individual patients with myasthenia gravis. Neurology 1981; 31: 220-4.

8 Gregerson DS, Abrahams IW, Thirkill CE. Serum antibody levels of uveitis patients to bovine retinal antigens. Invest Ophthalmol Vis Sci 1981; 21: 669-80.

9 Scadding JG, Mitchell DN. Sarcoidosis. London: Chapman and Hall, 1985: 414-43.

10 Lehner T, Almeda JD, Levinsky J. Damaged membrane fragments and immune complexes in the blood of patients with Behçet's syndrome. Clin Exp Immunol 1978; 34: 206-12.

11 Andrews BS, McIntosh J, Petts V, Avery R. Circulating immune complexes in retinal vasculitis. Clin Exp Immunol 1977; 29: 23-9.

12 Nussenblatt RB, Gery I, Ballintine EJ, Wacker WB. Cellular immune responsiveness of uveitis patients to retinal $\mathrm{S}$ antigen. Am J Ophthalmol 1980; 89: 173-9.

13 Dumonde DC, Kasp Grochowska E, Banga JP, et al. Autoimmune mechanisms in inflammatory eye disease. Trans Ophthalmol Soc UK 1985; 104: 232-8.

Accepted for publication 25 March 1987. 